\title{
Novel materials to enhance keratoprosthesis integration
}

\author{
S R Sandeman, R G A Faragher, M C A Allen, C Liu, A W Lloyd
}

\begin{abstract}
Background-The successful integration of keratoprostheses (KPros) within the cornea depends in part on peripheral host keratocyte adhesion to anchor the implant in place and prevent epithelial downgrowth. The following study incorporated different acrylate co-monomers with poly(hydroxyethyl methacrylate) (p(HEMA)) and measured the suitability of these materials as potential skirt materials in terms of their ability to enhance keratocyte adhesion to p(HEMA).

Methods-p(HEMA) hydrogels incorporating varying amounts of the acrylate co-monomers methacrylic acid (MA), 2-(dimethylamino)ethyl methacrylate (DEM), or phenoxyethyl methacrylate (PEM) were formed by free radical polymerisation. Keratocytes were seeded onto discs of each material and incubated at $37^{\circ} \mathrm{C}$ for 72 hours. Assays for viable cell adhesion were carried out. A viability/ cytotoxicity assay using solutions of calcein-AM $(0.5 \mathrm{mM})$ and ethidium homodimer-1 (EthD-1) (0.5 $\mu M)$ were used to measure viable and non-viable cell adhesion, respectively. An ATP assay was also used to quantify cell adhesion in terms of the amount of ATP present following lysis of adherent cells.

Results-The viability/cytotoxicity assays indicated that the incorporation of 15 mol\% of the co-monomer PEM or of 20 mol\% DEM increased cell adhesion to p(HEMA) by at least four times. The ATP assays confirmed the results for PEM but absorption of ATP to the DEM containing hydrogel indicated that the assay was not a suitable measure of cell adhesion to this material.
\end{abstract}

Biomaterials and Drug

Delivery Research Group, Department of Pharmacy and

Biomolecular

Sciences, Cockcroft

Building, University of

Brighton, Brighton,

East Sussex, BN2 4GJ

S R Sandeman

R G A Faragher

M C A Allen

A W Lloyd

Sussex Eye Hospital, Brighton, East Sussex C Liu

Correspondence to: S R Sandeman

s.sandeman@brighton.ac.uk

Accepted for publication 28 January 2000 chemical burns, and those Johnson syndrome or ocular pemphigoid. Various polymers have been investigated as potential keratoprosthetic materials. Those in clinical use tend to be poly(methylmethacrylate) (PMMA) based. ${ }^{2}{ }^{3}$ Additionally, osteoodonto-keratoprostheses (OOKPs), peripherally anchored by material derived from the patient's tooth, have shown some success. ${ }^{4}$ However, the long term success of purely plastic KPros is limited. All KPros currently available produce serious complications such as implant extrusion, retroprosthetic membrane formation, infection, and glaucoma. ${ }^{5}$ New KPro designs have sought to limit these complications by the incorporation of biocompatible materials which are conducive to implant integration within the host cornea. While the central optical cylinder should hinder cellular ingrowth, novel polymers are required for use as the peripheral skirt with properties that enhance host keratocyte adhesion and spreading. These will anchor the KPro to the surrounding tissue and induce controlled matrix remodelling so that the implant becomes an integral component of the cornea.

Numerous studies have considered the ability of various biomaterials to encourage cell adhesion and growth. The process is complex and involves the interaction of a number of factors including surface charge, wettability (hydrophilicity/hydrophobicity), porosity, and roughness. Cell adhesion and spreading tend to favour moderately hydrophilic materials which incorporate a positive charge and depend on initial protein absorption by the polymer. $^{6-8}$

Poly(hydroxyethyl methacrylate) (p(HEMA)) is well tolerated by biological tissues and has been used in a number of biomedical applications including the manufacture of soft contact lenses and intraocular lenses. ${ }^{8}$ However, fibroblast adhesion to untreated p(HEMA) is limited and cells do not tend to spread or exhibit normal morphological features. ${ }^{910}$ p(HEMA) hydrogels have been modified for use in a KPro prototype. The design was well tolerated on implantation into rabbit corneas and cell invasion into the pores of the material was observed. ${ }^{1112}$ However, tensile strength was limited because of the high percentage of water diluent required to achieve pores large enough for cell invasion. ${ }^{13}$ Further modifications may be necessary before $\mathrm{p}$ (HEMA) based designs, suitable for corneal implantation, are obtained. The cell adhesion characteristics of p(HEMA) have been improved by the incorporation of collagen ${ }^{14}$ and by the addition of hydrophobic caprolactone. ${ }^{15}$ Smetana et al found that monocyte adhesion was much greater onto co-polymers of $p$ (HEMA) and positively charged 2-(dimethylamino)ethyl 
Table 1 Polymer formulations

\begin{tabular}{lllll}
\hline Polymer & HEMA (g) & MA (g) & DEM (g) & PEM (g) \\
\hline p(HEMA) & 6.5 & - & - & - \\
0.5 mol\% MA, 99.5 mol\% p(HEMA) & 6.47 & 0.022 & - & - \\
1 mol\% MA, 99 mol\% p(HEMA) & 6.44 & 0.043 & - & - \\
$1.5 \mathrm{~mol} \%$ MA, 98.5 mol\% p(HEMA) & 6.4 & 0.065 & - & - \\
$0.5 \mathrm{~mol} \%$ DEM, 99.5 mol\% p(HEMA) & 6.47 & - & 0.039 & - \\
$1 \mathrm{~mol} \%$ DEM, 99 mol\% p(HEMA) & 6.4 & - & 0.079 & - \\
$20 \mathrm{~mol} \%$ DEM, 80 mol\% p(HEMA) & 5.2 & - & 1.57 & - \\
15 mol\% PEM, 85 mol\% p(HEMA) & 5.33 & - & - & 1.55 \\
\hline
\end{tabular}

methacrylate (DEM) (10 mol\%) when compared with that onto $\mathrm{p}$ (HEMA) or a copolymer of $\mathrm{p}(\mathrm{HEMA})$ and the negatively charged salt of methacrylic acid (MA) (3 $\mathrm{mol} \%)$. Bergethon et $a l^{10}$ had previously shown that the incorporation of either of the ionisable groups MA or DEM $(0.1 \%$ vol $)$ produced fibroblast spreading onto $\mathrm{p}$ (HEMA) hydrogels. Results indicate that the addition of charged monomers to $\mathrm{p}$ (HEMA) based hydrogels alters the cell adhesion and spreading characteristics of these polymers.

$\mathrm{p}$ (HEMA) has a number of extracellular matrix-like characteristics which make it suitable for consideration as a KPro material. It is well tolerated by biological tissues and will absorb water for hydration of the anterior cornea. In addition, its properties may be adapted for use as both the central, non-porous optic of the KPro and the peripheral, porous skirt. Problems of skirt adhesion to the core may be eliminated by the polymerisation of one component in the presence of the other, forming an interpenetrating meshwork between the two parts. ${ }^{15}$ This study sought to enhance the cell adhesion characteristics of a $p$ (HEMA) based skirt material by investigating the effect of incorporating aromatic, negatively charged, or positively charged acrylate based comonomers on keratocyte adhesion to $\mathrm{p}$ (HEMA).

\section{Materials and methods}

MATERIAL PREPARATION

Polymers were formed by free radical polymerisation using the initiator azo-isobutyronitrile (AIBN) and the cross linker ethylene dimethacrylate (EDMA). A range of $\mathrm{p}$ (HEMA) based materials was produced containing varying amounts of either the negatively charged co-monomer MA, the positive charged co-monomer DEM or the aromatic co-monomer phenoxyethyl methacrylate (PEM). The monomer solutions were weighed out in the amounts given in Table 1, mixed, and degassed by sonication. Initiator $(0.2$ $\mathrm{mol} \%)$ and cross linker $(0.5 \mathrm{~mol} \%)$ were added and the solution was injected into a polymerisation chamber. The polymerisation chambers consisted of two outer $6 \mathrm{~cm}^{2}$ glass plates separated by two sheets of commercially modified polyethylene terathyalate (PET) and a central hollow Teflon perimeter. The PET sheets have one hydrophobic surface and chambers were constructed so that the hydrophobic side was in contact with the polymer. The chambers were incubated at $60^{\circ} \mathrm{C}$ for at least 16 hours. Each polymer sheet was placed in a beaker of distilled water. The water was changed daily for 1 week to remove unpolymerised monomer and cross linker. Discs of each material were cut out and sterilised by autoclaving.

\section{VIABILITY/CYTOTOXICITY ASSAY}

A viability/cytotoxicity assay using calcein-AM and EthD-1 was used to quantify the effect of incorporating varying amounts of different co-monomers on the capacity of $\mathrm{p}$ (HEMA) to support viable cell growth. Calcein-AM is cleaved by cellular esterases present within viable cells to form a fluorescent green product which is membrane impermeable. EthD-1 is a fluorescent red marker which binds to nucleic acids and only passes through the compromised membrane of non-viable cells. Discs of each material were placed in the wells of a 24 well plate and incubated in phosphate buffered saline (PBS) for 24 hours at $37^{\circ} \mathrm{C}$. Pure $\mathrm{p}$ (HEMA) discs were used as a negative control and glass coverslips were used as a positive control. EK1.BR keratocytes established in the department ${ }^{16}$ were passaged by standard trypsinisation. Cells, at a concentration of $4 \times 10^{4}$, were pipetted onto each of the discs and the plates were incubated at $37^{\circ} \mathrm{C}$ in a 5\% carbon dioxide/air incubator for 72 hours. Following incubation, media were removed from the wells and calcein-AM (0.5 $\mathrm{mM})$ and EthD-1 (0.5 $\mu \mathrm{M})$ solutions were added. Discs were incubated for 10 minutes at room temperature then viewed under fluorescent microscope (Leitz). Calcein-AM positive, viable cells and EthD-1 positive, non-viable cells were counted in each of 30 fields for each material at wavelengths of $500 \mathrm{~nm}$ and $625 \mathrm{~nm}$ respectively at a magnification of $\times 400$. Results were tested for statistical significance using analysis of variance.

ATP ASSAY

The ATP assay was used as a second measure of viable cell adhesion to assess its suitability by comparison with the results of the viability assay above. The simple assay measures a light producing reaction between cell lysate ATP and substrate luciferin in the presence of luciferase and can be used as a measure of adherent biomass. The assay was carried out using ATP assay kits supplied by Labtech International and an Amerlite luminometer (Amersham International).

Keratocytes were incubated on discs of each material as previously described. Discs were washed in PBS and placed in a second 24 well plate so that only cells present on the materials were detected. A volume of $300 \mu \mathrm{l}$ of hypotonic lysis buffer was added to each well. The lysis buffer consisted of $5 \mathrm{ml}$ of $0.1 \mathrm{M}$ TRIS-acetate buffer, $3.2 \mathrm{ml}$ of a $0.1 \mathrm{M}$ solution of EDTA, and $16.8 \mathrm{ml}$ of sterile water. Plates were stored at $-20^{\circ} \mathrm{C}$ overnight. Following defrost $300 \mu \mathrm{l}$ of TRIS-acetate buffer was added to the cell lysate. A volume of $50 \mu \mathrm{l}$ of ATP monitoring reagent containing luciferol and luciferase was added to the wells of a 96 well microlitre plate and a background reading was taken. A volume of $150 \mu \mathrm{l}$ of diluted lysate covering the polymer discs was added to each well and a second luminescence reading was taken. All of the rea- 


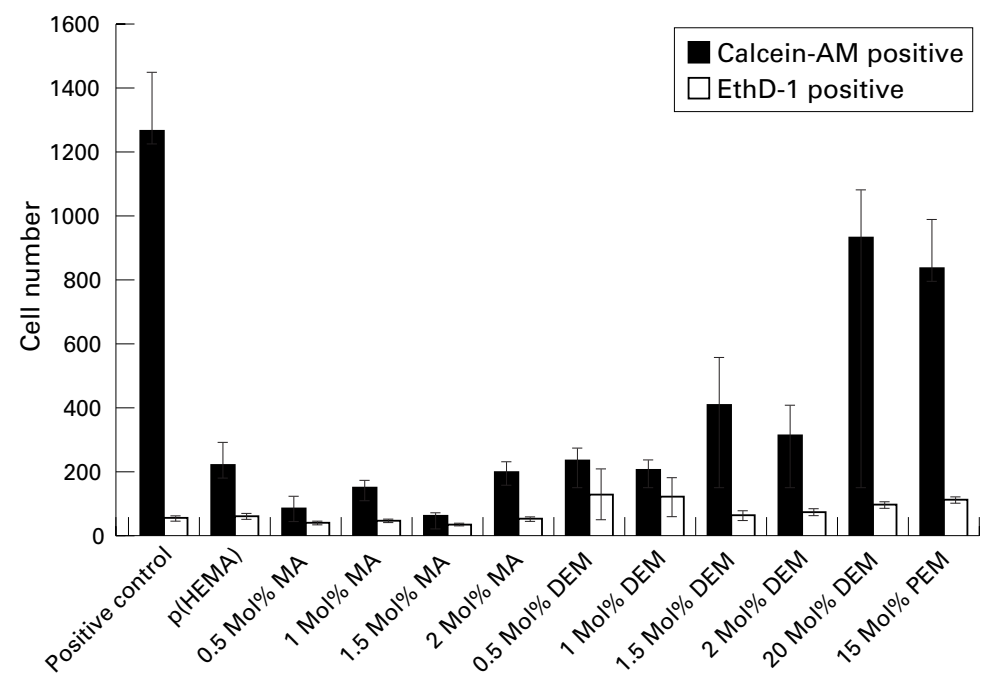

Polymer

Figure 1 Viability/cytotoxicity assay of p (HEMA) hydrogels incorporating negatively charged $M A$, positively charged DEM, or aromatic PEM co-monomers in varying concentrations (mean (SEM), $n=4$ ). Keratocytes were grown on discs of each material then assayed for adherent live cells with the fluorescent indicator calcein-AM $(0.5 \mathrm{mM})$ and for adherent dead cells with EthD-1 (0.5 $\mu \mathrm{M})$ using a fuorescent microscope. Cell adhesion was counted in 30 fields on each disc.

gents were kept on ice throughout the experiments. In order to confirm that ATP concentration and cell number are directly related an ATP assay using serially decreasing cell concentrations was carried out. Results were tested for statistical significance using analysis of variance.

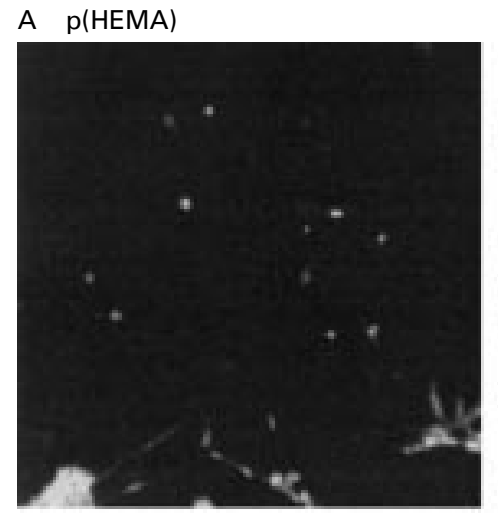

B Positive control

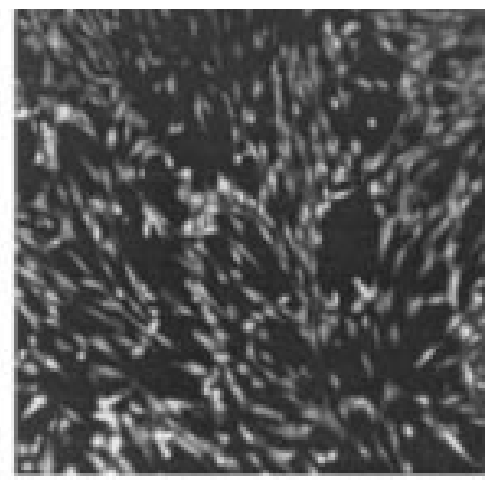

C $20 \mathrm{Mol} \% \mathrm{DEM}$

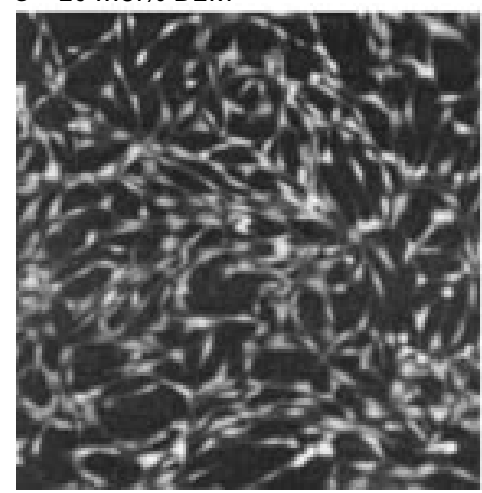

D $15 \mathrm{Mol} \%$ PEM

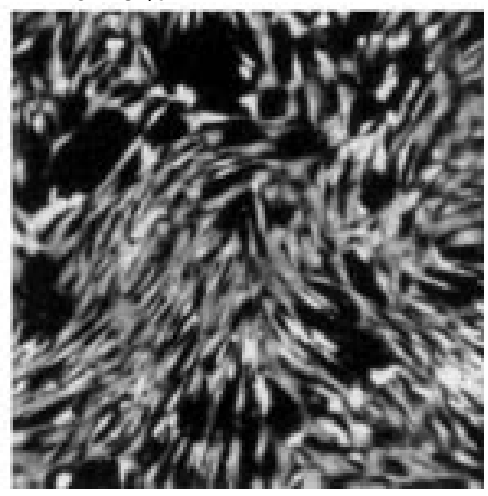

Figure 2 Calcein-AM fluorescent staining depicting keratocyte adhesion to $(A)$ p(HEMA), (B) positive control, (C) $20 \mathrm{~mol} \%$ DEM, (D) $15 \mathrm{~mol} \%$ PEM ( $\times 100$ magnification).

\section{Results}

VIABILITY/CYTOTOXICITY ASSAY

Figure 1 compares viable, calcein-AM positive cell adhesion with non-viable, EthD-1 positive cell adhesion to p(HEMA) hydrogels incorporating different co-monomers. A significant difference was observed in the ability of the different materials to enhance viable cell adhesion $(\mathrm{p}<0.05, \mathrm{n}=3)$. Addition of the negatively charged co-monomer MA did not improve the cell adhesion characteristics of the p(HEMA) hydrogels. The number of viable keratocytes present on the MA containing materials, indicated by calcein-AM positive counts, was similar to that of the pure $\mathrm{p}$ (HEMA) discs (Fig 1). Addition of the positively charged comonomer DEM did not improve the number of viable cells adhering to the materials when incorporated at low amounts (0.5-2 mol\%) but greatly enhanced viable cell adhesion to the p(HEMA) gels when incorporated at $20 \mathrm{~mol} \%$ (Fig 1). Incorporation of the aromatic comonomer PEM was also found to improve viable cell adhesion to the $\mathrm{p}$ (HEMA) hydrogels (Fig 1). Eth-D positive cell counts, indicating material cytotoxicity, were low for all materials. Keratocytes growing on the $20 \mathrm{~mol} \%$ DEM and $15 \mathrm{~mol} \%$ PEM containing p(HEMA) polymers exhibited normal spindle shaped fibroblast morphology while cells growing on the other materials tended to be rounded and clumped in places (Fig 2).

ATP ASSAY

The ATP assay of serially decreasing cell concentrations showed that ATP content increased with increasing cell concentration making it possible to use the assay as a measure of cell adhesion to the various polymers. While ATP content appeared to be directly related to cell number at low cell concentrations it levelled off at cell concentrations higher than $5 \times 10^{4}$ cells (Fig 3 ).

Figure 4 indicates significant differences in the amount of ATP lysed from cells adherent to the various $\mathrm{p}$ (HEMA) based materials suggesting differences in viable cell adhesion $(p<0.05, n=6)$. The results of the ATP assay of cell adhesion to the materials reflected those of the viability/cytotoxicity assay except for the results obtained for the $20 \mathrm{~mol} \%$ DEM containing polymers. Low levels of ATP activity, suggesting poor cellular adhesion, were found for the pure $\mathrm{p}$ (HEMA), MA containing polymers, and polymers containing low levels of DEM as observed in the previous assay (Fig 4). High levels of ATP activity were detected for the PEM containing discs as observed in the previous assay. However, the detection of ATP lysed from cells adherent to the $20 \mathrm{~mol} \%$ containing polymer discs was consistently low despite the observance, under light microscope, of high numbers of adherent cells on the discs before the assay and the results of the viability assays.

The possibility that the ATP released following lysis of adherent cells was being absorbed by the material was tested. One disc of each material was placed at $-18^{\circ} \mathrm{C}$ overnight with a known amount of the ATP standard in $400 \mu \mathrm{l}$ 


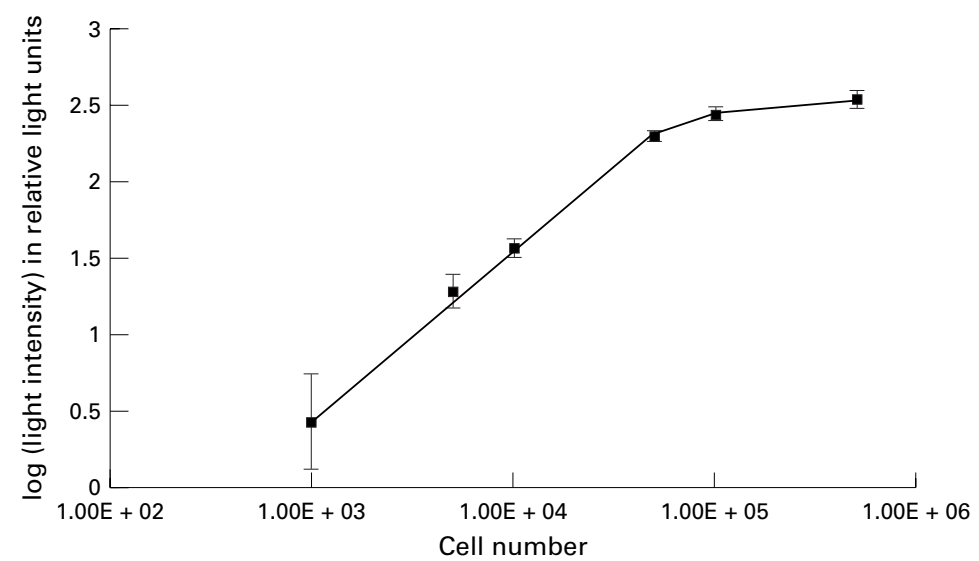

Figure 3 ATP assay of serially decreasing cell concentrations (mean (SEM), $n=9$ ). Cell suspensions were prepared containing four $\left(1 \times 10^{5}, 5 \times 10^{4}, 1 \times 10^{4}, 5 \times 10^{3}\right.$, and $\left.1 \times 10^{3}\right)$ cells. Cell suspensions were centrifuged at $400 \mathrm{~g}$ and the cell pellets were resuspended in $300 \mu \mathrm{l}$ of hypotonic lysis buffer. The cell lysate was stored at $-20^{\circ} \mathrm{C}$ for 16 hours. The cell lysate was diluted with TRIS-acetate buffer. A volume of $50 \mu \mathrm{l}$ of monitoring reagent and containing luciferin substrate and luciferase was added to the wells of a 96 well plate and $150 \mu l$ of cell lysate was added. The light emitted by the luciferase catalysed reaction of ATP with

luciferin was recorded using a luminometer.

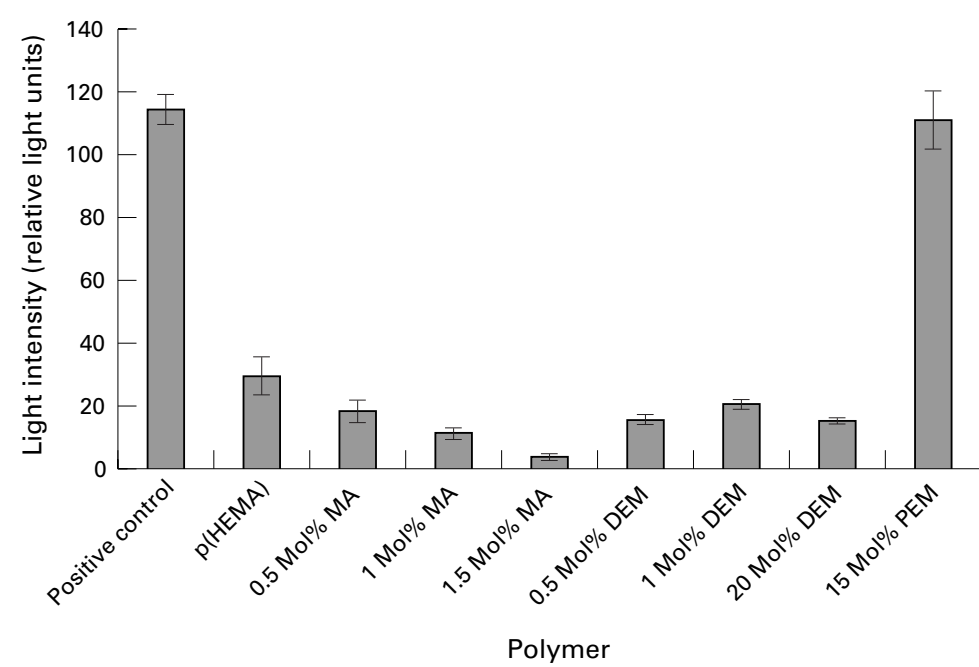

Figure 4 ATP assay quantifying cell adhesion to $M A, D E M$, and PEM containing $p(H E M A)$ hydrogels (mean (SEM), $n=6)$. Cells at a concentration of $4 \times 10^{4}$ were seeded onto triplicate discs of each material and incubated at $37^{\circ} \mathrm{C}$ for 72 hours. Discs were moved to a second 24 well plate and $300 \mu \mathrm{l}$ hypotonic lysis buffer was added. Plates were stored at $-20^{\circ} \mathrm{C}$ for 16 hours. Lysates were diluted 1:1 with buffer and $50 \mu \mathrm{l}$ of monitoring reagent was added to the wells of a 96 well microlitre plate. A volume of $150 \mu$ l of lysate was added to the monitoring reagent and light emission was measured.

TRIS-acetate buffer covering each disc. On comparison with a control with no material present the DEM discs produced a lower light intensity reading indicating that the DEM containing discs were absorbing some of the ATP released on lysis of the cells so that it was unavailable for the assay.

\section{Discussion}

As previously observed cell adhesion and spreading onto the $100 \% \mathrm{p}$ (HEMA) hydrogels was low. ${ }^{9}$ Keratocytes tended to be sparse and rounded on the discs although some cell spreading occurred in patches. The incorporation of $20 \mathrm{~mol} \%$ DEM but not low concentrations of MA or DEM improved cell adhesion and spreading onto p(HEMA) based hydrogels. Results were in agreement with those of Smetana et al but not with those of Bergethon et $a l^{10}$ who found that both DEM and MA
$(0.1 \%$ vol $)$ enhanced cell spreading. Since serum proteins tend to be negatively charged it is possible that protein absorption, which is required for cell adhesion, occurs more readily onto surfaces incorporating positive charge. ${ }^{17}$ The hydrophobic monomer PEM also appears to enhance cell adhesion and spreading onto $\mathrm{p}$ (HEMA) based hydrogels. Lydon et $a l^{6}$ suggest that moderation of $\mathrm{p}$ (HEMA) based hydrogel hydrophilicity by the incorporation of aromatic monomers enhances cell spreading and it may be that PEM alters the cell adhesion characteristics of $\mathrm{p}(\mathrm{HEMA})$ hydrogels in this way.

From the results of the ATP assay it is possible to confirm that the incorporation of PEM into a $\mathrm{p}$ (HEMA) hydrogel improves the ability of the material to maintain viable keratocyte growth. Although it is probable that the incorporation of $20 \mathrm{~mol} \%$ DEM also enhances p(HEMA) cell adhesion characteristics the absorption of ATP by the DEM containing polymers made it impossible to analyse this probability using the ATP assay. While the ATP assay gives some measure of the differences in cell adhesion to various polymers it was not found to be a sensitive or reliable assay and should be used in this context only in conjunction with the results of other assays. The potential for ATP absorption by the materials may produce errors. Also the light intensity readings vary considerably within a short time making comparison of results both within and between assays difficult. Variability is probably produced by the decay in activity of both the monitoring reagent and the lysed ATP which begins immediately following thawing.

In conclusion, the properties of $\mathrm{p}$ (HEMA) hydrogels may be modified by the incorporation of the positively charged acrylate comonomer DEM and by the hydrophobic acrylate co-monomer PEM to enhance cell adhesion and spreading. On this basis both materials may be considered as suitable KPro skirt materials. Further work to incorporate pores within the structure of these materials will enhance keratocyte colonisation for integration of the implant within the cornea.

1 Barnham J, Roper-Hall M. Keratoprosthesis: a long-term review. BrF Ophthalmol 1983;67:468-74.

2 Cardona, $\mathrm{H}$. The cardona experience: 40 years experience. Refract Corneal Surg 1991;7:468-71.

3 Dohlman C, Doane, $M$. Some factors influencing outcome after keratoprosthesis surgery. Cornea 1994;13:214-18.

4 Ricci R, Pecorella A, Ciardi A, et al. Strampelli's osteo-odonto-keratoprosthesis. Clinical and histological long-term features of three prostheses. $\mathrm{Br} f \mathrm{O}$ Ophthalmol 1992;76:232-4.

5 Hicks C, Chirila T, Dalton P, et al. Keratoprostheses: advancing toward a true artificial cornea. Surv Ophthalmol 1997;42:175-89.

6 Lydon M, Minnett T, Tighe B. Cellular interactions with synthetic polymer surfaces in culture. Biomaterials $1985 ; 6$ : 396-402.

7 Smetana K, Lukas J, Paleckova V, et al. Effects of chemical structure of hydrogels on the adhesion and phenotypic characteristics of human monocytes such as expression of falectins and other carbohydrate-binding sites. Biomaterials 1997;18:1009-14.

8 Kunzler J, McGee J. Contact lens materials. Chem Ind 1995; 16:651-5

9 Peluso G, Petillo O, Anderson J, et al. The differential effects of poly(2-hydroxyethyl methacrylate) and poly(2hydroxyethyl methacrylate)/poly(caprolactone) polymers on cell proliferation and collagen synthesis by human lung fibroblasts. F Biomed Mater Res 1997;34:327-36.

10 Bergethon P, Trinkaus-Randell V, Fransblau C. Modified hydroxyethylmethacrylate hydrogels as a modeling tool for 
the study of cell-substratum interactions. $\mathcal{F}$ Cell $S_{c i}$ 1989:92:111-21.

11 Crawford G, Constable I, Chirila T, et al. Tissue interaction with hydrogel sponges implanted in the rabbit cornea. Cornea 1993:12:348-57.

12 Crawford G, Chirila T, Vijayasekaran S, et al. Preliminary evaluation of a hydrogel core-and-skirt keratoprosthesis in the rabbit cornea. 7 Refract Surg 1996;12:525-9.

13 Hicks C, Chirila T, Clayton A, et al. Clinical results of implantation of the chirila keratoprosthesis in rabbits. $\mathrm{Br} f$ Ophthalmol 1998;82:18-25.
14 Civerchia-Perez L, Faris B, LaPointe G, et al. Use of collagen-hydroxyethylmethacrylate hydrogels for cell collagen-hydroxyethylmethacrylate hydrogels
growth. Proc Natl Acad Sci USA 1980;77:2064-8.

15 Hicks C, Lou X, Platten S, et al. Keratoprosthesis results in animals: an update. Aust NZ F Ophthalmol 1997;25(Suppl 1): $550-2$.

6 Dropcova S, Denyer S, Lloyd A, et al. A standard strain of human ocular keratocytes. Ophthalmic Res 1999;31:33-41.

17 Lee J, Khang G, Lee H. Interaction of cells on chargeable functional group gradient surfaces. Biomaterials 1997;18: $351-8$. 